\title{
WILEY-VCH
}

DOI: 10.1002/ ((please add manuscript number))

Article type: Full Paper

\section{Wearable Ring Resonator Antenna}

Bahareh Moradi*, Marc Martinez, Raul Fernández-García and Ignacio Gil

Bahareh Moradi, Raúl Fernández-García and Ignacio Gil are with the Department of Electronic Engineering, Universitat Politècnica de Catalunya, Terrassa (Barcelona) 08222, Spain

E-mail: bahareh.moradi@upc.edu

Keywords: Electro-textile, Metamaterial, Ring resonator, Specific absorption rate, Wearable Antenna

Abstract: This paper proposes a metamaterial wearable antenna. The proposed design is based on a transmission line coupled to a pair of double ring resonators (DRRs) on an e-textile substrate. The metallic antenna layer is fully embroidered providing both electromagnetic and mechanical good properties. The proposed design is validated by comparison of 3D electromagnetic simulations and measurements. The obtained results correspond to $\mathrm{S}_{11}<-29.5$ $\mathrm{dB}$ with a gain of $9.64 \mathrm{dBi}$ at resonance frequency and $93 \%$ efficiency covering a $160 \mathrm{MHz}$ bandwidth. In addition, the effect of environmental condition such as temperature and humidity on antenna is reported. Results show the resonance frequency level shift in several climatic conditions. Moreover, the specific absorption rate (SAR) to preserve the human body safety from radiation has been performed by means of numerical simulations, according to the international regulation. Experimental results confirm that the embroidered MTM antenna is a useful technique to control the propagation of signals on wearable applications.

\section{Introduction}

In the last decade the fast growing fields of wearable antennas have garnered worldwide research interest due to their applications in wireless systems for body area networks ${ }^{[1-5]}$. Since electronic textiles (e-textiles) are usually light weight, inexpensive, durable, zero maintenance and flexible (ability to bend, crumple), they are considered as a suitable material for wearable antennas in wireless systems. While these qualities provide advantages for a number of conventional textile applications, e-textiles are usually required to be robust and controllable against environmental conditions and, especially, to reduce their sensitivity to humidity and temperature. Furthermore, wearable antennas operate in close proximity to the human body. Therefore, the electrical and conformal effects due to the biological tissues (polar and lossy media at microwave frequencies) and body shape (bending impact) change the electrical performance of wearable antennas. ${ }^{[6,7]}$ Moreover, the reciprocal interaction between the antenna and human tissue makes the design of highly efficient wearable antennas with low specific absorption rate (SAR) an incentive.

Embroidery is the most advanced integration technique of electronic textile substrates, because embroidery machines allow repeatability, mass production of garments and customized designs in terms of thread distribution with a resolution in the order of $<1 \mathrm{~mm}$. ${ }^{[8]}$ Recently, a metamaterial (MTM) for miniaturized lightweight antenna was reported in, ${ }^{[9,10]}$ an embedded MTM wearable microstrip patch antenna was presented in ${ }^{[11]}$ and textiles MTMs for antenna applications have been reported in ${ }^{[12]}$. 


\section{WILEY-VCH}

In this paper a wearable MTM antenna based on embroidered double ring resonator (DRR) is presented. The antenna is implemented on a felt substrate that can be attached or integrated to clothing. The considered structure does not limit the possible antenna placements across the outfit. The degree of flexibility and one metallic layer design allows improving user's wearability and comfort. The antenna performance is also studied in detail and several on-body deployment scenarios in order to ensure safe SAR levels have been analyzed. Since most fabrics tend to absorb water and moisture or affected by temperature conditions, the proposed antenna is studied under demanding environmental scenarios.

The main novelty of the paper is based on the use of textile materials as substrate and metamaterial concept. The controllable electromagnetic properties of the proposed structure and the possibility of device flexibility and miniaturizability allows designing prototypes with improved performance and novel techniques, in comparison with conventional devices. This fact is crucial, since embroidery textile materials pose limitations to the achievable size, conductivity, substrate permittivity, substrate dielectric losses, and thickness of the prototypes, in comparison with standard printed circuit-board (PCB), whose fabrication techniques enable straightforward realization of the small conductive structures required as rigid circuit boards.

\section{Antenna design and experimental}

The proposed design layout is depicted in Fig. 1(a). The antenna topology consists of a pair of symmetrical double ring resonators (DRRs) with inner circle diameter $11=18 \mathrm{~mm}$ that are coupled to surrounding circle with diameter $12=28.2 \mathrm{~mm}$ and gap between them $\mathrm{g} 2=2 \mathrm{~mm}$. The gap between ring and host transmission line is $\mathrm{g} 1=1 \mathrm{~mm}$. The antenna is designed on a felt substrate with dielectric constant $\varepsilon r=1.2, \mathrm{~h}=1 \mathrm{~mm}$ thickness and loss tangent $\delta=0.0013$. The ground plane has been chosen as a homogeneous uniform commercial WE-CF adhesive copper sheet layer (constant thickness $t=35 \mu \mathrm{m}$ ), for simplicity. The felt substrate is chosen because of its intrinsic low loss tangent and low cost in comparison with other fabrics ${ }^{[13]}$. The DRRs structure behavior can be described by means of LC resonant parallel tanks. These DRRs are excited by the port feed through a capacitive coupling between the host line and ring resonators and capacitive coupling between rings resonator to each other. The DRRs shape of resonators has been chosen to maximize the capacitance of the LC resonant tank. The port feeding width is set to $\mathrm{wt}=4 \mathrm{~mm}$ and with a length approximately $13=\lambda / 4(77 \mathrm{~mm} @ \mathrm{f}=3.97 \mathrm{GHz})$.

For antenna fabrication, the embroidery machine Singer Futura XL550 is used. The selected conductor yarn corresponds to a commercial Shieldex 117/17 dtex 2-ply and it is composed by $99 \%$ pure silver plated nylon yarn $140 / 17$ dtex with a linear resistance $<30 \Omega / \mathrm{cm}$. The embroidered prototype is depicted in Fig. 1(b).

Due to the mechanical restrictions of the embroidery machine, the pattern was stitched with two types of threads. The sulky yarn is used as top thread and the conductive threads were used as bobbin threads. Also the default higher thread tension had to be lowered in order to maintain geometrical accuracy. The proposed design was embroidered with a satin pattern with $40 \%$ stitch density. The stitch spacing corresponds to the distance between two needle penetrations on the same side of a column.
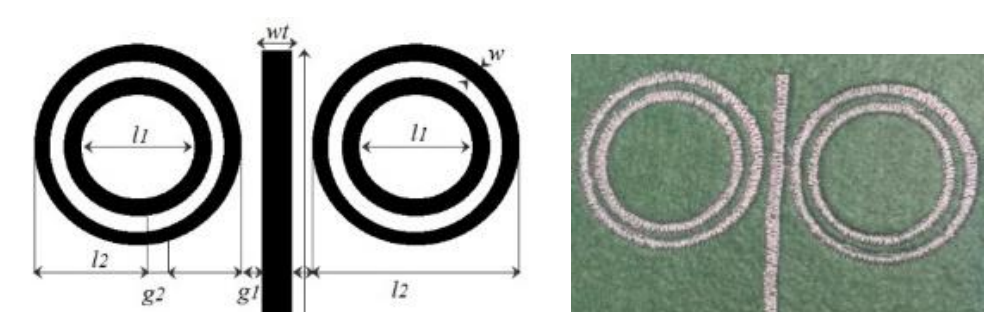
Figure 1. a) Geometrical sketch of the proposed e-textile MTM DRR antenna. b) Embroidered prototype on felt fabric.

The density determines the gap between stitches. For narrow columns, stitches are tight, thus requiring fewer stitches to cover the fabric. In areas with very narrow columns, less dense stitches are required because too many needle penetrations can damage the textile sample.

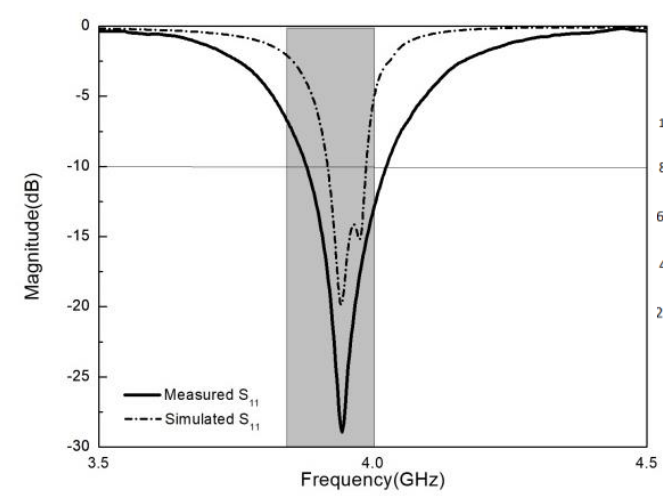

(a)

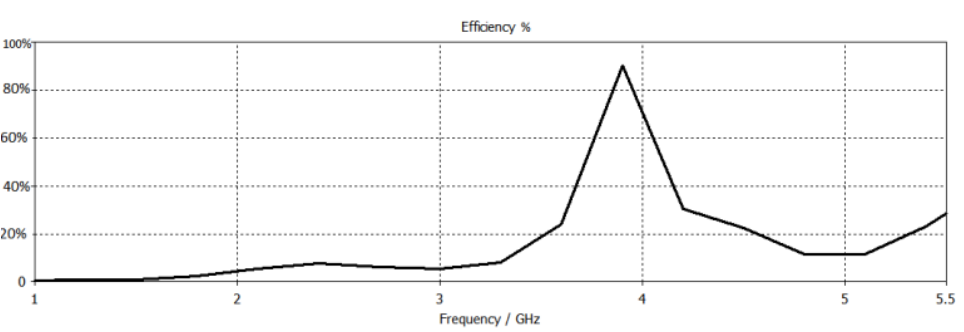

(b)

Figure 2. (a) S-parameter responses of the EM simulation and measurement of embroidered MTM antenna, (b) Efficiency Vs frequency of the proposed e-textile MTM DRR antenna.

Fig. 2(a) shows the measured and simulated values of the wearable antenna reflection coefficient (S11) and indicates a good agreement between them. The minimum measured $\mathrm{S}_{11}$ value is $-29.5 \mathrm{~dB}$ at $3.97 \mathrm{GHz}$. The $-10 \mathrm{~dB}$ fractional bandwidth (FBW) is $2.04 \%(3.84 \mathrm{GHz}-$ $4 \mathrm{GHz}$ ). The results show a good $50 \Omega$ antenna matching. The bandwith is enhanced by means of the double ring design due to the mutual coupling. The antenna radiation patterns were measured in an anechoic chamber. The free space antenna gain is $9.64 \mathrm{dBi}$ with $93 \%$ efficiency compared to $41 \%$ for individual SRR ${ }^{[14]}$ and $90 \%$ for the antenna with SRRs is reported in ${ }^{[15]}$. Fig. 2 (b) shows the variation of antenna radiation efficiency versus frequency, it can be seen that the radiation efficiency at $3.97 \mathrm{GHz}$ is $93 \%$. The experimental normalized radiation patterns compared against simulations are depicted in Fig. 3.

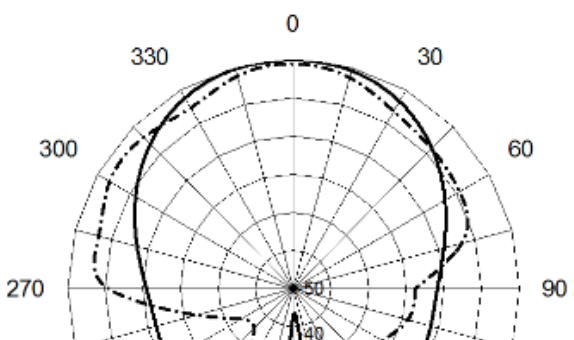

90

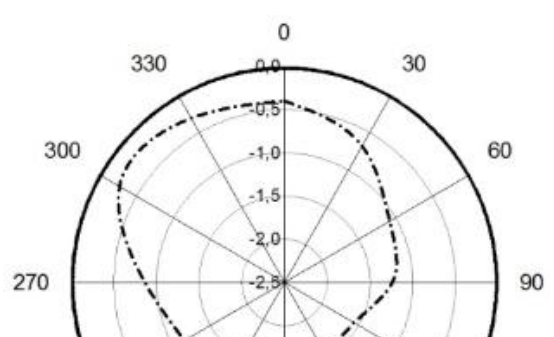




\section{WILEY-VCH}

Figure 3. Simulated and measured normalized radiation patterns of the e-textile MTM DRR antenna at $3.97 \mathrm{GHz}$, (a) XZ-plane $\left(\varphi=90^{\circ}\right)$. (b) YZ-plane $\left(\theta=0^{\circ}\right)$.

The current distribution and the real part of the magnetic field distribution generated at the resonance frequency are shown in Figures 4(a) and (b) respectively. The resonance enhancement is obtained by electrical coupling when the inner/outer ring combination is properly designed.

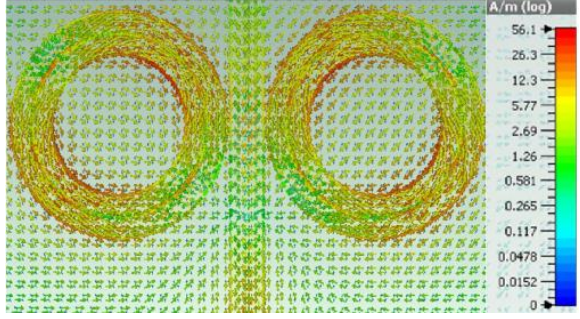

(a)

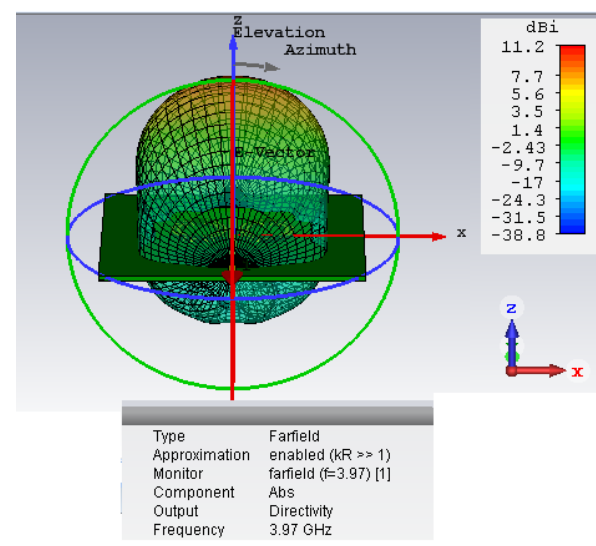

(c)

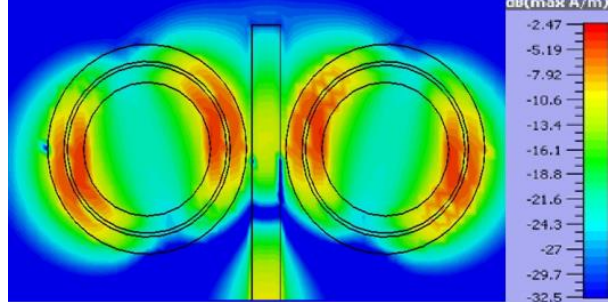

(b)

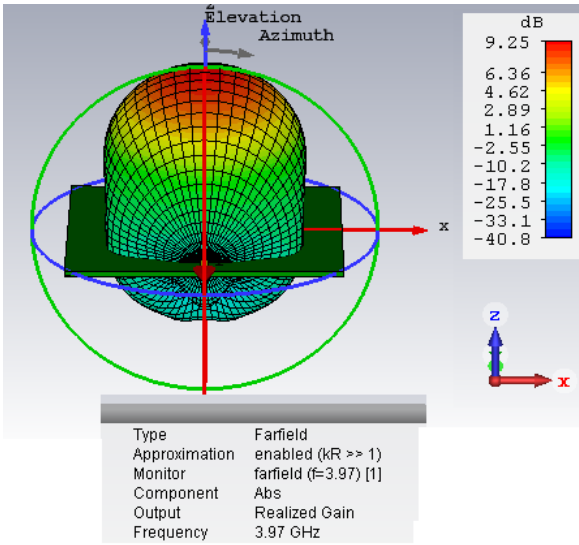

(d)

Figure 4. a) Current distributions and (b) magnitude field distribution for e-textile DRR antenna @ $3.97 \mathrm{GHz}$. (c) Simulated 3D view of the directivity radiation pattern at $3.97 \mathrm{GHz}$. (d) Simulated 3D view of the realized gain radiation pattern at $3.97 \mathrm{GHz}$.

Figures 4(c) and 4(d) depict the simulated radiation pattern of directivity and realized gain at $3.97 \mathrm{GHz}$ respectively. The material contribution to the radiation is silver with lossy metal and $6.3012 \mathrm{e} 7 \mathrm{~s} / \mathrm{m}$ electric conductivity.

\section{Environment and Body Impact}




\section{WILEY-VCH}

\subsection{Antenna under environmental conditions}

Electromagnetic properties of textiles can significantly change by environmental conditions such as humidity and temperature. For instance, the dielectric properties of the textile are increased by absorbing more moisture or increasing the temperature. ${ }^{[16]}$

The prototype was examined under several environmental conditions into a climatic chamber (CCK-40/125 model). The test started when the antenna sample was at $-20^{\circ} \mathrm{C}$ and the absolute humidity in the chamber was very low. The amount of water in the chamber remained small due to the relatively low temperature. After this, the amount of water in the chamber was gradually decreased, and the observed frequency followed closely to the changes in the relative humidity. The temperature was varied from $-20^{\circ} \mathrm{C}$ to $123^{\circ} \mathrm{C}$ and the extracted response frequency related to temperature changing is depicted in Fig. 5(a). The frequency response in different temperature with relative humidity $1 \%$ to $35 \%$ is illustrated in Fig. 5(b).

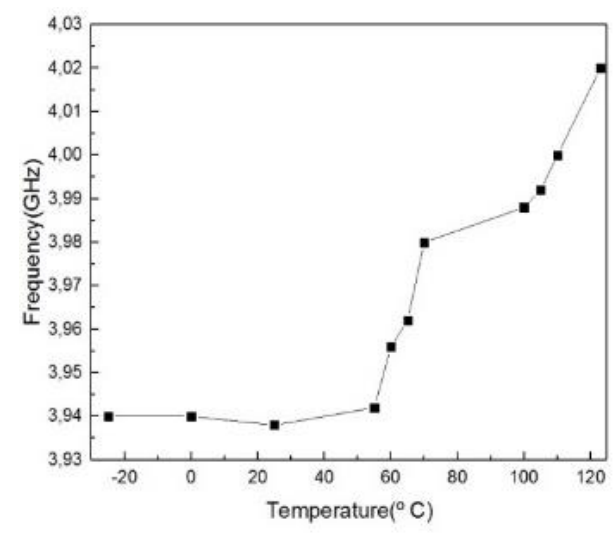

(a)

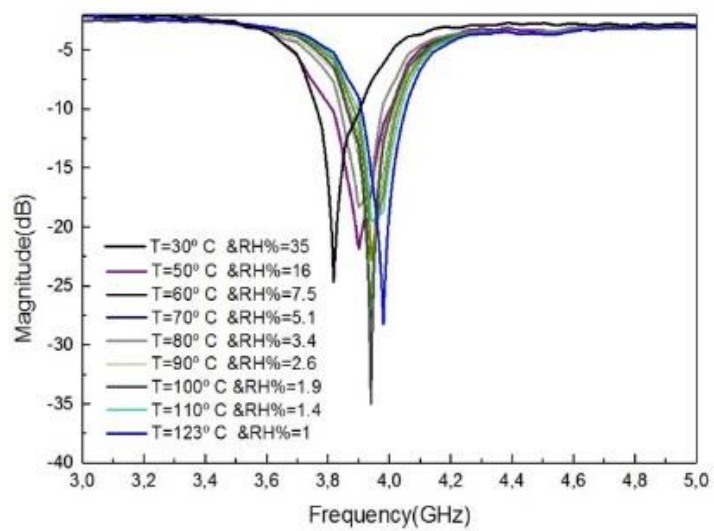

(b)

Figure 5.a) Effect of temperature on the antenna resonance frequency $(\mathrm{GHz})$. b) Measured reflection coefficient $\left(S_{11}\right)$ response by changing temperature and related humidity to that temperature.

The effect of temperature and related humidity determine the amount of vaporized and absorbed water in the chamber and the results indicate that as the amount of water in the chamber decreases, the reflection coefficient shifts from $3.84 \mathrm{GHz}$ at $30^{\circ} \mathrm{C}$ to higher frequency $(4.1 \mathrm{GHz}$ at $123^{\circ} \mathrm{C}$ ) with a sensitivity of $2.8 \mathrm{MHz} / \mathrm{C}^{\circ}$.

\subsection{SAR Validation}

SAR is a measure of the radiated power absorbed per unit mass in a human body tissue ${ }^{[17]}$. It is normally spatially averaged over a certain amount of exposed biological tissue. SAR is calculated using the root mean square (rms) of the electric field strength inside the human body, the conductivity, and the mass density of the tissue. The SAR limit recommended by European/Japan/China authorities is $2.0 \mathrm{~W} / \mathrm{kg}$ averaged over $10 \mathrm{~g}$ of actual tissue whereas in the United States/Canada/Korea the threshold corresponds to $1.6 \mathrm{~W} / \mathrm{kg}$ for averaged over a volume of 1 gram of tissue, for the body or head. In this work, modeling focuses on the realistic human voxel model Gustav from CST $2017^{[18]}$. Fig. 6 shows the detailed human voxel model and the wearable antenna located on the chest to enable antenna evaluation under bending radii. In this work the antenna is bent with a radius of $20 \mathrm{~mm}$ when placed on the chest. The input power to the antenna for SAR calculations is set at $0.05 \mathrm{~W}(\mathrm{rms})$, a typical maximum value for body area network applications, and their values were calculated based on the IEEE C95.1 standard, averaged over $1 \mathrm{~g}$ and $10 \mathrm{~g}$ of biological tissue. The 3D obtained results are depicted 


\section{WILEY-VCH}

in Fig. 6 (a), (b) respectively. The highest average SAR value in the band width for the model is shown in the table I. The results illustrated that the 1-g highest average SAR at the lower, center, and upper operating frequency bands of the antenna are 97.5\%, 68.2\% and 75\% respectively, lower than the CEU standard. Also for 10-g highest average SAR at the same operating frequency are $99.5 \%, 96 \%$ and $93 \%$ respectively, lower than CEU standard.

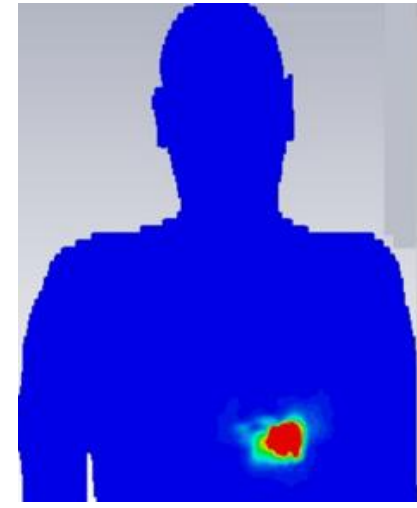

(a)
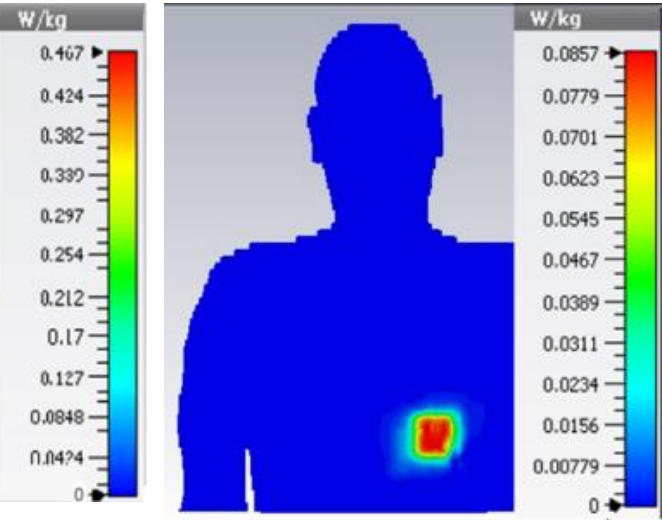

b)

Figure 6. a) SAR simulation setup on chest in Gustav model a) $1 \mathrm{~g}$ peak SAR b) $10 \mathrm{~g}$ peak SAR with averaging method IEEEC.95.3 and $0.05 \mathrm{~W}$ power.

Table 1. SAR peak values for the proposed antenna, averaged over (1 $\mathrm{g})$ and (10 $\mathrm{g})$ of biological tissue based on the IEEE C95.1 standard.

\begin{tabular}{|c|c|c|}
\hline Frequency $(\mathrm{GHz})$ & For 1 g SAR $(\mathrm{W} / \mathrm{kg})$ & For 10 g SAR (W/kg) \\
\hline high-frequency & 1.6 (based on CEU) & 2 (based on CEU) \\
\hline 3.84 & 0.04 & 0.012 \\
\hline 3.97 & 0.467 & 0.085 \\
\hline 4 & 0.395 & 0.147 \\
\hline
\end{tabular}

\subsection{Effect of bending}

In wearable systems, it is very difficult to keep the substrate on flat configuration all the time, especially when the prototype is made of textile materials and it is frequently bent due to human body morphology and movements. Therefore, it is necessary to investigate the prototypes performance characteristics under bending conditions. The $S_{11}$ parameters of the proposed etextile antenna under different bending conditions have been measured by taking into account typical bending human-shape radii $(10 \mathrm{~mm}<\mathrm{r}<45 \mathrm{~mm})$.

It is observed that due to bending, the equivalent length of the proposed design is modified and, hence, there are deviations in the resonance frequency. The evident from the experimental observations is shown in Fig.7. 


\section{WILEY-VCH}
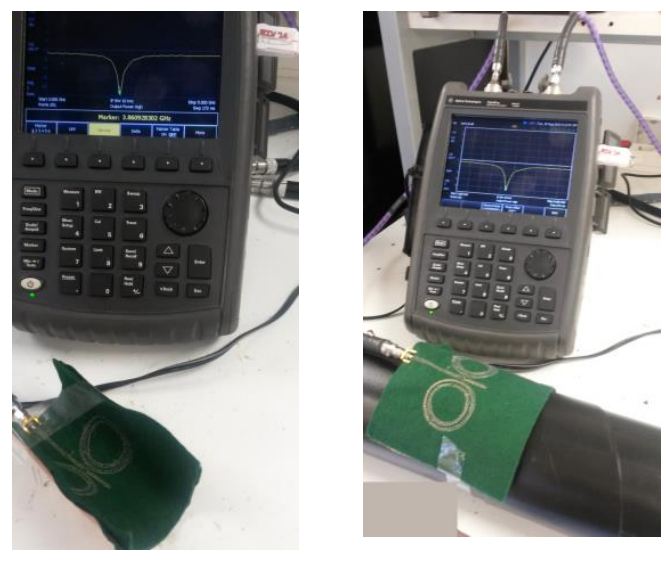

(a)

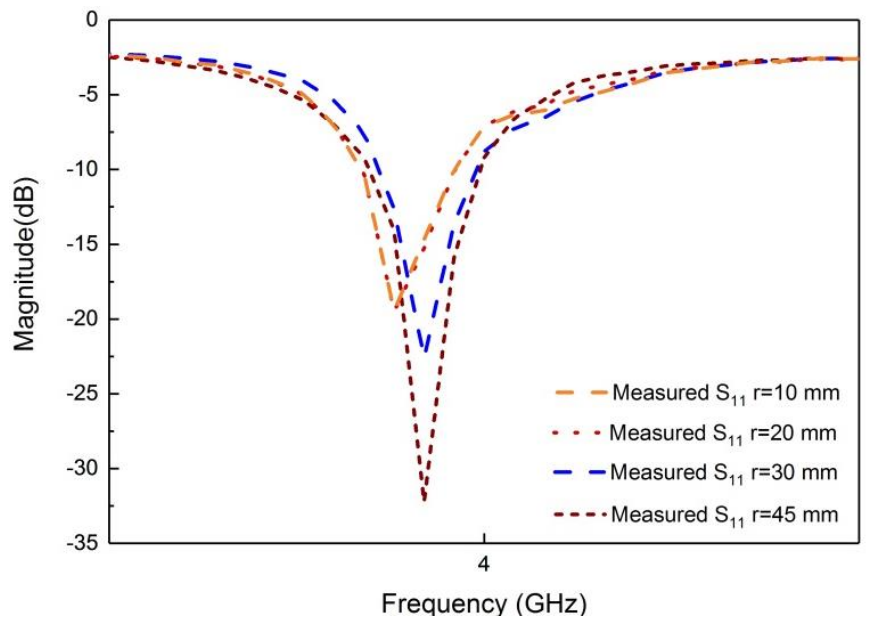

(b)

Figure 7. a) Photo of antenna bending with different curve angles. b) Resonance frequency of bending antenna with different radius: $10 \mathrm{~mm}, 15 \mathrm{~mm}, 20 \mathrm{~mm}, 30 \mathrm{~mm}$ and $45 \mathrm{~mm}$.

By changing the radius of bending from $10 \mathrm{~mm}$ to $45 \mathrm{~mm}$, the resonance frequency is shifted up to $40 \mathrm{MHz}$. From the S-parameters measured results shown in Fig.7 (b) we determine a high $\mathrm{S}_{11}$ peak of $-32 \mathrm{~dB}$ for a radius of $45 \mathrm{~mm}$ and one minimum $\mathrm{S}_{11}$ peak $(-18 \mathrm{~dB}$ at $3.88 \mathrm{GHz})$ with regard to the radius of $10 \mathrm{~mm}$.

\section{Conclusion}

In this paper the utilization of MTM antenna is presented for wearable applications. The proposed design is a fully embroidery conductive thread transmission line loaded with conductive yarn DRRs on felt fabric substrate. The comparison of full 3D electromagnetic simulations and measurements has been studied, achieving a significant degree of agreement. Also the effects of temperature and humidity changing of the manufactured prototype have been tested on chamber and quantified. The proposed antenna placed on the chest and SAR validation is obtained which the results were in the limitation range. Additionally, the effect of bending of the manufactured e-textile antenna has been tested. The validated results confirm that the embroidered MTM antenna is a useful technique to control the propagation of signals on wearable applications.

\section{Acknowledgements}

This work was supported by the Spanish Government MINECO under project TEC201679465-R

\section{References}




\section{WILEY-VCH}

[1] A dual-mode single-band wearable microstrip antenna for body area networks, IEEE

Antennas and Wireless Propagation Letters, C. Mendes, C. Peixeiro, pp 3055 - 3058, vol.16, 2017.

[2] Impact of body and clothing on a wearable textile dual band antenna at digital television and wireless communications bands, IEEE Transactions on Antennas and Propagation, D. L. Paul, H. Giddens, M. G. Paterson, G. S. Hilton, J. P. McGeehan “, pp. 2188 - 2194, vol.61, 2013.

[3] A textile antenna for off-body communication integrated into protective clothing for firefighters, IEEE Transactions on Antennas and Propagation. C. Hertleer, H. Rogier, L. Vallozzi, L. V. Langenhove, pp. 919-925, vol.57, 2009.

[4] Wearable button antenna for dual-band WLAN applications with combined on and offbody radiation patterns, IEEE Transactions on Antennas and Propagation. H. Xiaomu, S. Yan, G. A. E. Vandenbosch, vol. 65, pp. 1384-1387, 2017.

[5] Dual polarized textile patch antenna for integration into protective garments, IEEE Antennas Wireless Propag, L. Vallozzi, H. Rogier, and C. Hertleer, Lett, vol. 7, pp. 440-443, 2008.

[6] Textile Antennas: Effects of Antenna Bending on Input Matching and Impedance Bandwidth, IEEE Aerospace and Electronic Systems Magazine, P. Salonen., Rahmat-Samii, Y., Vol. 22, No. 12, pp. 18 - 22, Dec. 2007.

[7] Wearable Antennas for FM Reception, European Conference on Antennas and Propagation, T. Kellomaki. J. Heikkinen, M. Kivikoski, Nov. 6-10, 2006.

[8] Embroidery and Related Manufacturing Techniques for Wearable Antennas, challenges and opportunities, Electronics A. Tsolis, Whittow, W.G., Alexandridis, A.A., pp.314-338, 2014. 


\section{WILEY-VCH}

[9] Functionalized Metamaterials Enable Frequency and Polarization Agility in a Miniaturized Lightweight Antenna Package Advanced Electronic Materials, C.P.Scarborough, D.H.Werner, D.E.Wolfe, vol. 2, 2016.

[10] Peiwei Chen, Liang Peng, Aiting Wu, Gaofeng Wang, Rendering wide impedance band of ESA made of SRRs, Electronic Letters, Vol. 52 No. 19 pp. 1582-1584, 2016

[11] Metamaterial embedded wearable rectangular microstrip patch antenna, International Journal of Antennas and Propaga, Special issue on Wearable Antennas and Systems, Hindwai Publication Corporation, J. G. Joshi, Shyam S. Pattnaik, and S. Devi, ID. 974315, pp.1-9, doi: 10:1155/2012/974315, 2012.

[12] Fabric antennas integrated with metamaterials, Metamaterials, R. Seager, A. Chauraya, and J. Vardaxoglou, vol. 1, p. 533535, 2008.

[13] Embroidery and Related Manufacturing Techniques for Wearable Antennaschallenges and opportunities, Electronics, A. Tsolis. W.G. Whittow, A.A. Alexandridis; 3: 314-338, 2014.

[14] Liang Peng, Peiwei Chen, Aiting Wu \& Gaofeng Wang, Efficient Radiation by Electrically Small Antennas made of Coupled Split-ring Resonators, Scientific Reports, 6, $33501,2016$.

[15] Albert Sabban, Small Wearable Antennas for Wireless Communication and Medical Systems DOI: 10.1109/RWS.2018.8304974, IEEE Radio and Wireless Symposium, 2018. [16] Measurement of dielectric properties of textile materials and their applications. Indian Journal of Fibre and Textile Research, Bal, K., \& Kothari, V. 34, 191-199, 2009.

[17] Specific absorption rate (SAR) evaluation of textile antennas, IEEE Antennas Propag. Mag P. J. Soh et al, 2015.

[18] https://www.cst.com/2017. 